\title{
INSTABILITIES IN PHOTOIONIZED INTERSTELLAR GAS
}

\section{Edvige Corbelli and Andrea Ferrara}

\author{
Preprint n. 8/95
}

\begin{abstract}
Osservatorio Astrofisico di Arcetri, Largo E. Fermi 5, I-50125 Firenze (Italy)
\end{abstract}

To appear in The Astrophysical Journal (July 10, 1995). 


\begin{abstract}
We present a linear analysis of acoustic and thermo-reactive instabilities in a diffuse gas, photoionized and heated by a radiation field, cooled by collisional excitation of hydrogen and metal lines. The hydrogen recombination reaction has a stabilizing effect on the thermal mode found by Field (1965) since the condensation instability occurs in a narrower region of the parameter space and grows on longer time scales due to its oscillatory character. This effect is stronger when the mean photon energy is not much larger than the hydrogen ionization energy. Moreover, for fixed values of thermal pressure and photoionization rate, there are thermo-reactive unstable equilibria for which no transition to a stable phase is possible. By extending our analysis of the thermo-reactive modes to the nonlinear regime we show that when no phase transition is possible the medium evolves through a series of nonequilibrium states characterized by large amplitude, nonlinear periodic oscillations of temperature, density and hydrogen ionization fraction. We find also unstable acoustic waves which, for solar metal abundances, are the fastest growing modes in two temperature intervals: around $T \sim 100 \mathrm{~K}$ and $T \sim 8000 \mathrm{~K}$ (i.e., cold and warm phase, respectively), independent of the mean photon energy. Possible implications for the interstellar medium and intergalactic medium are briefly outlined.

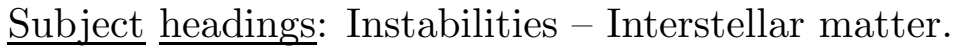




\section{INTRODUCTION}

Diffuse gas irradiated by an external radiation field is commonly found in many astrophysical environments. Often the radiation field constitutes the main energy input for the medium through ionization processes. For example, the cosmic UV background is responsible for heating and ionizing the Ly $\alpha$ clouds (Ikeuchi \& Turner 1991; Miralda-Escudé \& Ostriker 1992; Charlton et al.1993; Kulkarni \& Fall 1993) and the outer disks of galaxies (Corbelli \& Salpeter 1993a,b; Maloney 1993; Dove \& Shull 1994). Photoionization models of the Broad Line Region have been successful in the interpretation of AGN spectra (Kwan \& Krolik 1981; Collin-Souffrin 1990). Finally, in our Galaxy, examples of objects in which photoionization processes play a primary role are the High Velocity Clouds (HVCs) (Songaila et al.1989; Ferrara \& Field 1994), and the extended electron layer (Reynolds 1993, Domgörgen \& Mathis 1994). In this paper we shall be interested in mostly neutral gas, and our results do not apply to objects like bright HII regions, where cooling processes are likely be different from the ones considered here.

Interstellar gas very often tends to develop a multi-phase structure caused by ongoing thermal instabilities (Field 1965; Field et al.1969; McKee \& Ostriker 1977) and evidence of a two-phase medium has been found also in some of these photoionized diffuse objects (see for example Ferrara \& Field 1994 and references therein, Krolik et al.1981). In addition, gas at high galactic latitude and in outer disks of galaxies shows broad HI emission profiles. Broad features might originate from a turbulent medium or from a kinetic temperature. In both cases the phenomenon is of interest since in outer disks there is no evidence of substantial star forming activity, which could be responsible for the turbulence, and temperatures derived for a uniform warm medium are often thermally unstable against temperature and density perturbations (Dickey, Murray, \& Helou 1990; Verschuur \& Magnani 1994). In this respect, we believe it is important to consider the effect of ionization processes on the thermal instability and other unstable modes in diffuse media.

A new mode, in addition to the usual thermal (or condensation) mode and to the wave (or acoustic) modes, is found when the time dependence of ionization is taken into account. We

shall refer to this new mode and to the thermal one together as thermo-reactive modes. The ionization and recombination reaction has a stabilizing effect on the thermal instability of a pure hydrogen gas which is collisionally ionized (Defouw 1970; Ibañez \& Parravano 1983; Yoneyama 1973). The open question is whether the same effect arises in a photoionized gas 
or in gas where cooling is dominated by metal line excitation. Goldsmith (1970) worked out a first approach to the problem by studying the transition between the thermally unstable and stable regimes for a metal cooled medium, photoionized and heated by an external radiation field. Flannery \& Press (1979) pointed out the presence of unstable acoustic modes in the thermally stable cold phase of this gas, but in general our knowledge of the properties and existence of acoustic and thermo-reactive instabilities is still fragmentary. Photoionized gas in many objects is mostly warm or in a multi-phase structure. In this paper we will examine in detail both thermo-reactive and acoustic modes over a wider range of temperatures, metallicity and photon fluxes. Furthermore, the analysis of thermoreactive instabilities will be extended to the nonlinear regime. We neglect the magnetic field; when present, it implies a reduction of the thermal conduction coefficient and of the growth rate of the perturbations in the direction perpendicular to the field lines.

The plan of the paper is as follows: in $\S 2$ we present the basic equations for the equilibrium and stability analysis; in $\S 3$ we concentrate on the properties of acoustic and thermoreactive modes. Detailed numerical results of the linear analysis are shown in $\S 4$, and $\S 5$ contains the results of the nonlinear analysis for the thermo-reactive modes. Possible applications and a brief summary of the most interesting results are given in $\S 6$.

\section{PHYSICAL PROCESSES AND BASIC EQUATIONS}

We consider an ideal homogeneous gas with metallicity $Z$, and ratio of specific heats $\gamma$. We assume that hydrogen is the only reacting species. The basic equations are

$$
\begin{gathered}
\frac{d \rho}{d t}+\rho \nabla \cdot \mathbf{v}=0 \\
\rho \frac{d \mathbf{v}}{d t}+\nabla p=0 \\
N_{0} \frac{d x}{d t}-I(x, \rho, T)=0, \\
\frac{N_{0}}{\gamma-1}(1+x) k_{B} \frac{d T}{d t}+N_{0}\left(\frac{k_{B} T}{\gamma-1}+\chi\right) \frac{d x}{d t}+\mathcal{L}(x, \rho, T)-\frac{p}{\rho^{2}} \frac{d \rho}{d t}=0, \\
p=N_{0} \rho(1+x) k_{B} T,
\end{gathered}
$$


where the various symbols are defined in Table 1. Equations (2.1) and (2.2) express mass and momentum conservation; equation (2.3) describes the change of the hydrogen ionization fraction, $x$. In the energy equation (2.4) the first two terms describe changes of the internal energy due to variations of the temperature and of the degree of ionization, respectively. The binding energy $\chi$ of electrons to $\mathrm{H}$ atoms has been explicitly included in the internal energy, therefore we shall also take it into account in the heat-loss function $\mathcal{L}$. In this paper we neglect magnetic fields and heat conduction and use $\gamma=5 / 3$.

The steady state equilibrium is characterized by the values of $\rho_{0}, x_{0}, T_{0}, p_{0}$ and by $v_{0}=0$; as usual $\mathcal{L}\left(x_{0}, \rho_{0}, T_{0}\right)=I\left(x_{0}, \rho_{0}, T_{0}\right)=0$. We first investigate the response of the system to infinitesimal perturbations of the form

$$
\delta f=f_{1} e^{n t+i \mathbf{k} \cdot \mathbf{r}}
$$

where $n=n_{r}+i n_{i}$ is the growth rate; thus $n_{r}>0\left(n_{r}<0\right)$ means that the mode is unstable (stable). The perturbed variables $\rho, v, x, T, p$ will be then written as $f_{0}+f_{1}$. Linearizing the system (2.1)-(2.5), we obtain the equivalent homogeneous system $M \zeta=0$, where the vector $\zeta$ and the matrix $M$ are

$$
\begin{gathered}
\zeta \equiv\left(\begin{array}{c}
\rho_{1} \\
v_{1} \\
x_{1} \\
T_{1} \\
p_{1}
\end{array}\right) ; \\
M \equiv\left(\begin{array}{ccccc}
n & i k \rho_{0} & 0 & 0 & 0 \\
0 & \rho_{0} n & 0 & 0 & i k \\
-I_{\rho} & 0 & N_{0} n-I_{x} & -I_{T} & 0 \\
-\frac{1}{\rho_{0}} & 0 & -\frac{1}{\left(1+x_{0}\right)} & -\frac{1}{T_{0}} & \frac{1}{p_{0}} \\
-\frac{p_{0}}{\rho_{0}^{2}} n+\mathcal{L}_{\rho} & 0 & N_{0}\left(\frac{3}{2} k_{B} T_{0}+\chi\right) n+\mathcal{L}_{x} & \frac{3}{2} N_{0}\left(1+x_{0}\right) k_{B} n+\mathcal{L}_{T} & 0
\end{array}\right)
\end{gathered}
$$

The above system has a non-trivial solution only if $|M|=0$, which gives the following characteristic equation for the growth rate $n$

$$
\sum_{j=0}^{4} a_{j} n^{4-j}=0
$$

where

$$
a_{0}=1 ; \quad a_{1}=c_{s}\left[K_{T}+(1+\tilde{\chi}) J_{T}-J_{x}\right] ; \quad a_{2}=c_{s}^{2}\left[K_{x} J_{T}-K_{T} J_{x}+k^{2}\right]
$$




$$
\begin{aligned}
& a_{3}=k^{2} c_{s}^{3}\left[\frac{3}{5}\left(K_{T}-K_{\rho}\right)+\left(1+\frac{3}{5} \tilde{\chi}\right)\left(J_{T}-J_{\rho}\right)-\left(J_{x}-J_{\rho}\right)\right] \\
& a_{4}=\frac{3}{5} k^{2} c_{s}^{4}\left[\left(K_{x}-K_{\rho}\right)\left(J_{T}-J_{\rho}\right)-\left(K_{T}-K_{\rho}\right)\left(J_{x}-J_{\rho}\right)\right] .
\end{aligned}
$$

In the definition of $K_{T}, K_{\rho}, K_{x}, J_{T}, J_{\rho}, J_{x}$ given in Table 1 we have introduced some characteristic time scales of the system: three cooling times $\tau_{T}, \tau_{\rho}, \tau_{x}$, and three reactive times $\bar{\tau}_{T}, \bar{\tau}_{\rho}, \bar{\tau}_{x}$. Various combinations of these time scales, together with the dynamical time $\tau_{d}$, characterize the evolution of the perturbed gas. The isochoric regime holds when the cooling and reactive times are much smaller than the dynamical time, while in the isobaric regime the dynamical time is the shortest timescale. The general solution of a fourth degree polynomial as in eq. (2.7) is known and can be obtained analytically; however, due to its complexity, it is not very handy. A criterion, derived by Hurwitz (see for example Aleksandrov et al.1963), provides a necessary and sufficient condition, in terms of the $a_{j}$, for the real part of the roots to be all negative, i.e., stable modes for our case. This criterion has been applied by Yoneyama (1973) to a fourth degree dispersion relation similar to that given by equation (2.7) both for small and large wavenumbers.

We consider a medium heated and ionized by a background radiation field of mean photon energy $E_{0}$ (weighted by the photoionization cross section) and ionization rate $\xi_{0} . E$ and $\xi$ are the values of these quantities at a given optical depth, and in this paper we neglect any radiative transfer effects. We are interested in low density gas with equilibrium temperature $30<T<30,000 \mathrm{~K}$; we neglect three-body recombinations, and radiative recombinations are considered to levels $\geq 2$ (on-the-spot approximation) with a rate coefficient $\alpha$. Collisional ionization can become important at high temperature and we have adopted the ionization rate $\gamma_{c}$ given by Black (1981). Secondary electrons are included according to the results of Shull \& Van Steenberg (1985); the number of secondary electrons $\phi$, and the heat released for each photoionization, $E_{h}$, are functions of the hydrogen ionization fraction and of the photon energy $E$. We examine models for different values of the metallicity $Z$, ranging from the pure hydrogen case $(Z=0)$ to metal rich systems with $Z=2$. The adopted ionization-recombination function $I$ and heat-loss function $\mathcal{L}$ per gram are the following

$$
I=N_{0}^{2} \rho\left\{\frac{(1-x)}{N_{0} \rho} \xi(1+\phi)-x^{2} \alpha+(1-x) x \gamma_{c}\right\}
$$




$$
\mathcal{L}=N_{0}^{2} \rho\left\{(1-x) Z \Lambda_{H Z}+x Z \Lambda_{e Z}+(1-x) x \Lambda_{e H}+x^{2} \Lambda_{e H^{+}}-\frac{(1-x)}{N_{0} \rho} \xi\left[E_{h}+(1+\phi) \chi\right]\right\}
$$

The energy losses due to recombinations require some care in the calculation when we include $\chi$, the binding energy of the electrons in the ground state $\mathrm{n}=1$. The final expression for $\Lambda_{e H^{+}}$in the on-the-spot approximation is

$$
\Lambda_{e H^{+}}=\left(E^{t h}+\chi\right) \alpha
$$

$E^{t h}$ is the thermal energy lost by recombination. Using equations (2.10) and (2.3) it would be possible to rewrite the energy equation (2.4) only in terms of thermal energies if collisional ionizations are negligible, but we prefer this form for the sake of clarity and completeness. The functions $E^{t h}$ and $\alpha$ have been adapted from Seaton (1959)

$$
\begin{gathered}
\alpha=\frac{2.06 \times 10^{-11}}{\sqrt{T}}\left(0.50 \ln \Theta+0.47 \Theta^{-1 / 3}-0.32\right) \mathrm{cm}^{3} \quad \mathrm{~s}^{-1} \\
E^{t h} \alpha=7.2 \times 10^{-30} T \sqrt{\Theta}\left(0.5 \ln \Theta+0.64 \Theta^{-1 / 3}-0.821\right) \text { erg } \mathrm{cm}^{3} \mathrm{~s}^{-1}
\end{gathered}
$$

Note that even if we include recombinations to the ground state in the above expression, the cooling is considerably larger than the one used by Defouw (1970, cfr. eq. [22]) for recombinations to the $\mathrm{n}=1$ level only: at $T=4000 \mathrm{~K}$, for example, the cooling due to recombinations to all levels is a factor 2.4 larger than that due to recombination to $n=1$ only. Moreover, if the on-the-spot approximation is not used, and ionization by an external radiation field is considered, Lyman continuum photons should be added in the ionization equation.

It turns out that in our models cooling due to recombinations is ineffective compared to the other types of cooling. The expressions for energy losses due to collisional excitation of line radiation have been taken from Dalgarno \& McCray (1972), except for the fine structure excitation of CII and OI by atomic hydrogen impact, for which we used the results of Launay \& Roueff (1977), and for the Ly $\alpha$ excitation of neutral hydrogen for which we refer to Spitzer (1978). The metal species considered are CII, SiII, FeII, SII, OII, NI, OI; we have assumed that the ionized to neutral ratios for $\mathrm{O}$ and $\mathrm{N}$ are the same as for hydrogen to account for charge exchange effects. 


\section{ACOUSTIC AND THERMO-REACTIVE INSTABILITIES}

In Figure 1 we present the equilibrium curves in the plane $p_{0} / \xi-T_{0}$ for four different values of the metallicity $Z=0,10^{-3}, 0.5,1$ and three values of the mean photon energy $E=15,40,100 \mathrm{eV}$ taken as representative of gas at different optical depth. The dashed line segments indicate unstable equilibria; i.e., at least one of the four modes has positive $n_{r}$ for some $k$. The specific nature of the various unstable modes will be discussed later on in this Section.

For the pure hydrogen case $(Z=0)$, the equilibrium curves are monotonically decreasing functions of temperature and are generally stable. The cooling function is dominated by Ly $\alpha$ line emission excited by e-H impact; free-free cooling is negligible at these temperatures as well as recombination radiation losses since for a photoionized medium these are important only at temperatures $T \sim E_{h} / k_{B}$ (see also Section 4.3). As the mean energy of the penetrating photons increases, the heat deposited per photoionization gets larger and a higher density is needed to balance the heating via collisional cooling. Note that for the case $Z=0$, becuse of the lack of metal coolants, below $6500 \mathrm{~K}$ no realistic equilibria exist. Due to the low efficiency of the e-H impact cooling below $6500 \mathrm{~K}$, even a small amount of metals, as for $Z=10^{-3}$, makes the metal cooling dominate at these temperatures, and a three-phase medium is found for $E \gg 13.6 \mathrm{eV}$. For photon energies slightly above $13.6 \mathrm{eV}$ each photoionization releases no more than a few $\mathrm{eV}$ and an enhancement of the metal cooling has two consequences: (a) the maximum possible equilibrium gas temperature decreases; (b) $p_{0} / \xi$ is a monotonically decreasing function of $T_{0}$, i.e., multi-phase equilibria cannot exist.

A consequence of the Field (1965) isobaric instability criterion $(\partial \mathcal{L} / \partial T)_{p}<0$, is that thermally unstable regions are characterized or by a positive slope of the $p_{0}-T_{0}$ curve. This follows from the following relation

$$
\left(\frac{d \ln p}{d \ln T}\right)_{\mathcal{L}}=-\frac{T}{\rho} \frac{(\partial \mathcal{L} / \partial T)_{p}}{(\partial \mathcal{L} / \partial \rho)_{T}}
$$

being $(\partial \mathcal{L} / \partial \rho)_{T}>0$ for most astrophysical applications. When $\mathcal{L}$ depends on $x$, as well as on $\rho$ and $T$, there is an additional term proportional to the temperature derivative of the ionization fraction

$$
\left(\frac{\partial \mathcal{L}}{\partial T}\right)_{p}=\left(\frac{\partial \mathcal{L}}{\partial T}\right)_{\rho, x}-\frac{\rho}{T}\left(\frac{\partial \mathcal{L}}{\partial \rho}\right)_{T, x}+\frac{\partial x}{\partial T}\left[\frac{\partial \mathcal{L}}{\partial x}-\frac{\partial \mathcal{L}}{\partial \rho} \frac{\rho}{1+x}\right]_{\rho, T}<0
$$


or, in our notation,

$$
\left(K_{T}-K_{\rho}\right)+\frac{\partial x}{\partial T}\left(K_{x}-K_{\rho}\right)<0
$$

this criterion reduces to the Field criterion when $x$ is kept constant; otherwise one cannot read the stability properties directly off the slope of the equilibrium curve.

The low photon energy cases in Figure 1 exemplify very well the above point since for $Z=1$ there are negative slope parts of the equilibrium curve that show unstable isobaric modes, contrary to what would be expected applying Field criterion. As we raise the photon energy, the slope of $p_{0} / \xi$ becomes positive for a certain range of temperatures. For photon energies above $100 \mathrm{eV}$ the curves are similar to that of $E=100 \mathrm{eV}$, but they are shifted towards larger values of $p_{0} / \xi$.

\subsection{Dispersion curves: $n_{r}(k)$}

The hydrodynamical system described by eqs. (2.1)-(2.5) admits four different normal modes when the physical variables are perturbed as in equation (2.6). The characteristic equation is a fourth degree polynomial with real coefficients (eq. 2.7) and has zero or an even number of real roots. In other words, if there are complex roots, they should be complex conjugate pairs. In addition to the three modes found by Field (1965), a new mode is introduced by the ionization equation (2.3). Investigation of this additional "reactive mode" is important for two reasons: first, because it may initiate an instability driven by ionization changes; second, because the characteristics of the thermal mode might change. The nature of the modes can be easily determined by studying their behavior in the small and large wavenumber limits and they can be classified as two acoustic (hereafter denoted by WW) and two thermo-reactive (TR) modes. The solution in the limit of small (isochoric) and large (isobaric) wavenumbers can be written as:

$$
k \rightarrow \infty\left\{\begin{array}{l}
n_{1}=i c_{s} k-\frac{b_{1}-b_{3}}{2}, \\
n_{2}=-i c_{s} k-\frac{b_{1}-b_{3}}{2}, \\
n_{3}=\frac{1}{2}\left[-b_{3}+\sqrt{b_{3}^{2}-4 b_{4}}\right], \\
n_{4}=\frac{1}{2}\left[-b_{3}-\sqrt{b_{3}^{2}-4 b_{4}}\right] ;
\end{array}\right.
$$




$$
k \rightarrow 0\left\{\begin{array}{l}
n_{1}=i c_{s} k \sqrt{\frac{b_{4}}{b_{2}}}-\frac{c_{s}^{2} k^{2}\left(b_{2} b_{3}-b_{1} b_{4}\right)}{2 b_{2}^{2}}, \\
n_{2}=-i c_{s} k \sqrt{\frac{b_{4}}{b_{2}}-\frac{c_{s}^{2} k^{2}\left(b_{2} b_{3}-b_{1} b_{4}\right)}{2 b_{2}^{2}},} \\
n_{3}=\frac{1}{2}\left[-b_{1}+\sqrt{b_{1}^{2}-4 b_{2}}\right], \\
n_{4}=\frac{1}{2}\left[-b_{1}-\sqrt{b_{1}^{2}-4 b_{2}}\right],
\end{array}\right.
$$

where the coefficients $b_{i}$ are independent of $k$ and are related to $a_{i}$ 's of eq.(2.7) through a combination of powers of $c_{s} k \equiv \tau_{d}^{-1}$

$$
\begin{gathered}
a_{1}=b_{1} ; \quad a_{2}=b_{2}+c_{s}^{2} k^{2} ; \\
a_{3}=b_{3} c_{s}^{2} k^{2} ; \quad a_{4}=b_{4} c_{s}^{2} k^{2} .
\end{gathered}
$$

The solutions $n_{1}$ and $n_{2}$ represent the acoustic modes: if not overdamped, they oscillate with group velocity $d n_{i} / d k \sim c_{s}$. In the isochoric regime propagation can be slower than the sound speed by a factor $\left(b_{4} / b_{2}\right)^{1 / 2}$, which in all the cases studied never differs from unity by more than a factor of 10. These oscillatory solutions are always stable for $k=0$. The thermo-reactive modes correspond to solutions $n_{3}$ and $n_{4} ; d n_{i} / d k=0$ for any $k$ and therefore they do not propagate. Since in our case $b_{1}>0, b_{2}>0$, each equilibrium discussed in this paper will be thermo-reactively stable at small $k$. In this limit thermoreactive modes are fast (in the sense that they are damped in a time much smaller than the dynamical time) and obey the reduced characteristic (isochoric) equation $n^{2}+b_{1} n+b_{2}=0$. In the opposite limit (large $k$ ) thermo-reactive modes grow or damp on timescales much longer than the dynamical time, and satisfy the reduced (isobaric) equation $n^{2}+b_{3} n+b_{4}=$ 0 .

In many cases a continuous dispersion curve $n_{r}(k)$ corresponds to an acoustic mode in the isochoric regime, but to a thermo-reactive mode in the isobaric one. The nature of each solution in this case is not conserved when passing from the isochoric to the isobaric regime, and modes might not be identifiable at some intermediate wavenumber.

We will illustrate in Figure 2 three different dispersion curves for a particularly representative case, namely $E=100 \mathrm{eV}, Z=1$, since these summarize all the possible behaviors of $n_{r}(k)$ in unstable regions. Numerical values refer to $N_{0} \rho=1 \mathrm{~cm}^{-3}$; since wavenumber 
and growth rates scale linearly with the density, extension to other density values is obtained multiplying both $k$ and $n$ by $N_{0} \rho$. All three curves represent WW modes for small $k$ (i.e., $k \lesssim 10^{-18} \mathrm{~cm}^{-1}$ ) and TR modes for large $k$; TR (WW) modes in the isochoric (isobaric) regime are stable for all three cases. In the isochoric regime WW modes are oscillatory and unstable, implying, according to eq. $(3.2), b_{1} b_{4}>b_{2} b_{3}$. For $T_{0}=120 \mathrm{~K}, n_{r}$ has a maximum at $\log k=-17.8$ followed by an abrupt break leading to stable TR modes. The minimum growth time for WW is $2.5 \times 10^{7}\left(N_{0} \rho\right)^{-1}$ yr (the curve shown has been multiplied by a factor of 10). Increasing the temperature, TR modes become unstable for large $k$, and the curve for $T_{0}=160 \mathrm{~K}$ represents a transition case between $\mathrm{TR}$ stable and unstable regimes. Except for this transition region, when TR modes are unstable, their growth rate is usually larger than the WW one (WW modes remain unstable anyway), and therefore they represent the most dangerous modes for the system. A more typical dispersion curve for unstable WW and TR modes is that shown for $T_{0}=200 \mathrm{~K}$; as for the usual thermal instability $n_{r}$ grows monotonically with $k$ and the curve flattens out as $k \rightarrow \infty$. The effect of thermal conduction in this case would be to stabilize very large wavenumber perturbations

\subsection{Nature and properties of the modes}

It is possible to gain some qualitative understanding of the main results from an analysis of the dispersion curves in the limit of small and large $k$. The physical explanation of the acoustic instability at small $k$ is the following. When a fluid element is compressed and then rarefied by an acoustic wave there will be a net energy flow into the wave, destabilizing it, if more energy is gained by the gas during the compression phase than is lost in the expansion phase (Field 1965). For long enough wavelengths, the dependence of the ionization on $\rho$ and $T$ brings this about as follows. For long enough wavelengths, the ionization time is shorter than the period of the wave, so we are in ionization equilibrium at all times. During the compression phase $\rho$ increases, increasing the recombination rate. $T$ may increase somewhat also, but not enough to affect the recombination rate significantly. Hence, $x$ decreases in the compression phase; since the cooling rate is proportional to $x$, it decreases, and there is therefore a net gain of energy from the external radiation field. In the expansion phase, $x$ increases, and there is a net heat loss, but that is secondary, as shown by Field (1965), so the net effect of the ionization degree of freedom is to destabilize long waves (Flannery \& Press 1979). If the wavelength is short enough so that the ionization 
time is longer than the wave period, $x$ does not change, and the wave mode becomes stable. As a consequence, the existence of the critical wavenumber for WW stabilization is fixed by the condition that the dynamical time is roughly equal to the ionization time. At that value of $k$ the oscillation rate ( $\propto k$ in the entire range) becomes much shorter than the ionization time and stiffening of the fluid caused by ionization is no longer possible. Unstable acoustic waves are not found to be oscillatory in the usual thermal instability study (see Fig. 1 of Field [1965]), but they are overdamped if the dependence of the cooling function on density arises from two-body collisions.

For $T_{0}=120 \mathrm{~K}$ Figure 2 shows that the system is acoustically unstable but thermoreactively stable; however, except for the effects of ionization, it would be to thermally unstable at this temperature. For the equilibrium conditions we have considered ionization is in fact a stabilizing agent with respect to thermal instability. In addition it may introduce an oscillatory part in the growth rate, and in this case we shall refer to them as overstable modes. These findings have important implications and represent completely new features introduced by ionization.

In the isobaric limit, we have seen at the beginning of this section that the system (2.1)(2.5) reduces to $n^{2}+b_{3} n+b_{4}=0$ for thermo-reactive modes. This can be interpreted as the characteristic equation of a damped oscillator described by

$$
\frac{d^{2} y}{d t^{2}}+b_{3} \frac{d y}{d t}+b_{4} y=0
$$

where $y$ is the displacement of $x$ or $T$ from their equilibrium values, $b_{3}$ is the damping coefficient and $\sqrt{b}_{4}$ is the natural frequency of the oscillator. A necessary condition both for stability (see also Hurwitz's criterion) and for the motion to be oscillatory is $b_{4}>0$. Moreover, if $b_{4}>0$ stability depends only on the sign of $b_{3}$; since from eq. (2.7) the terms $J_{T}-J_{\rho}$ and $-\left(J_{x}-J_{\rho}\right)$ in $b_{3}$ are positive, they both tend to make $b_{3}>0$, hence to stabilize TR modes.

To understand the physical reason for the stabilization effect suppose that the cooling is dominated by electron-metal impacts. Since for large $k$ the perturbation is isobaric, a decrease in $T$ corresponds to an increase in the density, and, if the initial state is thermally unstable, $\rho$ will increase even further. However, on the ionization time scale (if not too large, see below) $x$ will react to the increased $\rho$ by decreasing. If $K_{x}-K_{\rho}>0$ the cooling will decrease, and as a consequence the temperature will raise up again, trying to counteract the instability. The condition $K_{x}-K_{\rho}>0$ is always verified when cooling is 
dominated by electron-metal impact and $x \ll 1$, since in that case the heat-loss function depends linearly on $x$, however the end result of the perturbation depends also on time scales. From the expression of $b_{3}$ it can be seen for example that stabilization by ionization is not effective for oscillatory modes if the characteristic cooling time scale $\tau_{\rho} \tau_{T} /\left(\tau_{T}-\tau_{\rho}\right)$ is shorter than the reactive timescales $\bar{\tau}_{\rho} \bar{\tau}_{T} /\left(\bar{\tau}_{T}-\bar{\tau}_{\rho}\right)$, and $\bar{\tau}_{\rho} \bar{\tau}_{x} /\left(\bar{\tau}_{\rho}-\bar{\tau}_{x}\right)$.

\section{NUMERICAL RESULTS: LINEAR PHASE}

In this Section we present results of the linear stability analysis. There are three free parameters in our model, namely, the mean photon energy, $E$, the metallicity of the gas, $Z$, and the photoionization rate $\xi$ (or, equivalently, $T$ ). Over a wide range of wavenumbers and for each value of $E, Z, \xi$, we solve numerically eq. (2.7) obtaining the four eigenvalues and the instability domains.

If for given value of the parameters each eigenvalue has a positive real part, $n_{r}<0$,for any $k$, the equilibrium is stable. When this does not happen it is necessary to inspect the dispersion curves $n_{r}-k$ to understand the specific nature of the instabilities since isobaric and isochoric limits are known (eq.(3.1) and (3.2)). In addition, dispersion curves provide information on the maximum positive real part of the growth rates, $n_{m}$, which indicates the most dangerous instability for the system. Results are shown in Figure 3(a) both for low-metallicity systems and metal-rich ones. If more than one mode is unstable, we plot only $n_{m}$ of the fastest one. If $n_{r}$ does not grow monotonically with $k$ towards some positive constant but presents a maximum located at some wavelength $k_{m}$, in Figure 3(b) we plot $k_{m}$. Both wavenumber and growth rates refer to the case $N_{0} \rho=1 \mathrm{~cm}^{-3}$ and scale linearly with the density.

Figure 4 summarizes the extension of stable and unstable regions in the plane $E-T$ for several values of $Z$. Dotted areas indicate where only acoustic instabilities exist and therefore these are the fastest modes. For low metallicity $Z \lesssim 0.1$ such regions do not exist; they first appear at low $E$. For $Z \geq 1$ two separate branches which are only acoustically unstable are found; one at low $T$ and one at high $T$, each of which widens as $Z$ increases. For $E$ just above $13.6 \mathrm{eV}$ equilibria are more often thermally stable but acoustically unstable. In the dashed regions the equilibrium is unstable for any $k$ : except close to the low and high temperature borders next to the acoustic unstable regions, the 
most unstable modes are the thermo-reactive ones for large $k$. Therefore we shall refer to these regions as thermally unstable regions. The extension of such regions is not a monotonic function of $Z$ : for the cases shown its minimum extension is for $Z=0.5$. At these intermediate $Z$ the reactive mode has the strongest stabilizing effect, while at high $Z$ it stabilizes the thermal mode but it produces acoustically unstable modes.

\subsection{Low-metallicity systems}

We examine here the stability of systems with a low metal content; in particular we select the two values $Z=0$ and $Z=10^{-3}$. In the absence of metals the stability analysis shows that the hydrogen gas is stable for perturbations of all wavenumbers, independently of the ionizing photon energy and of the intensity of the ionizing photon flux.

A small amount of metals in the system changes the shape of the equilibrium curve, as already mentioned when discussing Fig. 1 , and an unstable mode appears for $100 \lesssim T \lesssim 6000$ $\mathrm{K}$. The mode is unstable at all wavenumbers and changes from an overdamped acoustic instability in the isochoric limit ( $\operatorname{small} k$ ) to a thermo-reactive one for large $k$ where the largest growth rate occurs; $n_{i}=0$, for any $T, k, E$.

\subsection{High-metallicity systems}

The nature of the instability and the unstable temperature range in high metallicity systems depend more strongly on the mean photon energy and the metal abundance. As already mentioned in Section 3, there is one common feature to unstable modes: either they are thermo-reactively stable at small scales, $\lambda \lesssim 1 /\left(N_{0} \rho\right)$ pc, but acoustically overstable at larger scales or they are unstable at all scales, acoustically at large scales but thermo-reactively at small scales, where they mostly have the fastest growth rates.

For low values of $E$ (see Figure 4) the gas is thermo-reactively stable for $Z=0.5$ or lower but there are two overstable acoustic waves $\left(n_{r}>0, n_{i} \neq 0\right)$ over a wide range of $T$. For example, in the case of $E=15 \mathrm{eV}$, Figure 3 shows that the shortest growth time for these unstable acoustic modes is for $T_{0} \simeq 500 \mathrm{~K}$ and equals $2 \times 10^{6} /\left(N_{0} \rho\right)$ yr for $Z=0.5$ and $10^{5} /\left(N_{0} \rho\right)$ yr for $Z=1$. The scales corresponding to these growth times are $\sim 0.5 /\left(N_{0} \rho\right)$ pc and $\sim 0.1 /\left(N_{0} \rho\right)$ pc respectively. These parameters for $Z=1$ yield an optical depth $\sim 1.5$ : this means that the mean free path of photons is shorter than the wavelength of the maximum growth rate $\left(2 \pi / k_{m}\right)$ and therefore it is likely that the instability grows slowly on the mean free path scale, $N_{0} \rho \sigma(E)$, if this is still unstable $(\sigma(E)$ 
is the hydrogen photoionization cross section). By increasing the mean photon energy the gas tends to become unstable over all wavenumbers and the maximum growth rate is for TR modes with $\lambda<\left(0.01 / N_{0} \rho\right)$ pc. For $E \gg 13.6 \mathrm{eV}, Z$ needs to be larger than 0.5 to have some range of temperatures where only acoustic modes are unstable. For these energies Fig. 4 shows that instabilities occurs independently of $E$, and therefore at any depth inside the cloud.

The range of temperatures for which the gas is thermo-reactively unstable becomes wider as we increase $Z$ from 0.5 to 1 or 2 , independently of $E$. The growth time of unstable modes decreases with the gas metal content; for example Figure 3 shows that when $Z$ changes from 0.5 to 1 and $E \gg 13.6 \mathrm{eV}$, the growth time becomes smaller by a factor of 3 . Notice that when TR modes are overstable the maximum growth rate decreases: in Figure 3 for $Z=0.5$ and $E=40 \mathrm{eV}, n_{m}$ shows a dip in the interval $1000 \lesssim T \lesssim 2000 \mathrm{~K}$, where there are two overstable modes. The frequency of oscillation $n_{i}$ is equal or higher than $n_{r}$. For $Z \sim 1$ or larger and temperatures close to the region where only WW modes are unstable, TR modes are coupled and oscillatory. The coupling between TR modes tends to vanish as we increase $E$ or decrease $Z$.

\subsection{Comparison with previous results}

In order to better understand the results discussed here it is instructive to compare them with the ones obtained in previous works on the subject.

Field (1965) analyzed the thermal instability in a gas neglecting fractional ionization changes. This implies that there are only three modes, two waves and a non-oscillatory "condensation" mode. If we apply Field's criterion to our model, we find that there is an unstable non-oscillatory mode which represents an acoustic overdamped wave at low $k$, while it becomes an unstable condensation mode for large $k$ where the growth rate of the mode is much larger. The main differences from Field's analysis for a perturbation of the fractional ionization are: $(a)$ the temperature range in which a gas is thermally stable is wider and for low $E$ the thermal instability is suppressed for any $T$ (see Fig. 3-4); (b) stability is no longer connected with the shape of the $p_{0} / \xi$ curve; $(c)$ acoustic waves as well as thermal modes are often oscillatory; $(d)$ there are regions where the gas is thermally stable but nevertheless acoustic waves are isochorically unstable.

Flannery \& Press (1979) already found the presence of unstable acoustic waves in cold thermally stable regions. They studied the stability of a photoionized gas with a single 
metal coolant excited by electron impact only and under the assumption $x \ll 1$. When the metal coolant is CII, $Z=1$, and $E \simeq 40 \mathrm{eV}$, they find that unstable acoustic waves exist only where the gas is thermally stable and $30<T<90 \mathrm{~K}$. With the more complete cooling function adopted here (eq. 2.9) and dropping the condition $x \ll 1$, we find (see Figure 4 for the case $Z=1$ ) that: (a) the region $90<T<160 \mathrm{~K}$ is no longer thermally unstable since ionization is able to suppress the condensation mode for some equilibrium, leaving acoustic waves as the only relevant unstable modes of the system; $(b)$ the acoustically unstable region is located at $60<T<160 \mathrm{~K} ;(c)$ a new acoustically unstable region arises in the warm phase for $T \simeq 8000 \mathrm{~K}$, a fact that may have several interesting astrophysical consequences.

Defouw (1970) studied the stability of a pure hydrogen gas cooled by recombinations only and including the reactive mode. He mainly focused on a collisionally ionized gas, dealing with the photoionized case only briefly. Apart from an incorrect recombination coefficient (see $\S 3$ ), he stated that photoionized gas is thermally unstable if the mean energy of the photoelectron is less than the mean kinetic energy of the recombining electron. In our notation, this condition translates into $E-\chi<k T$, and therefore cases with $E \gg 13.6 \mathrm{eV}$ should be stable. However, if heating is provided by photons with $E \gg 13.6 \mathrm{eV}$ the system cannot be in equilibrium if the only cooling is provided by radiative recombinations; thus, according to Defouw, the gas should be always unstable (Yoneyama 1973 has studied a model similar to the one by Defouw, reaching similar conclusions). Inclusion of e-H collisional excitation cooling allows several stable equilibrium temperatures. A detailed model for a collisionally ionized pure hydrogen plasma, with the inclusion of e-H cooling, has been studied by Ibañez \& Parravano (1983) who obtain a modified instability criterion with respect to Defouw (the gas is stable in the two temperature ranges $T<8000 \mathrm{~K}$ and $13000 \mathrm{~K}<T<19000 \mathrm{~K}$ while for Defouw the gas is stable for $T<17000 \mathrm{~K}$ ). However these authors have not studied the stability for the case of a photoionized, metal cooled gas and therefore their results are not directly comparable with ours. 


\section{NONLINEAR ANALYSIS}

The aim of this Section is to investigate the nonlinear development of the TR instability in the large $k$ limit by solving the complete set of hydrodynamic equations for the isobaric case numerically. We choose an equilibrium state $\zeta_{0} \equiv\left(T_{0}, x_{0}\right)$ (which also fixes the value of $p / \xi$ uniquely) and impose a nonlinear perturbation $\delta \equiv(\delta T, \delta x)$; the resulting perturbed state $\zeta=\zeta_{0}+\delta$ is then used as the initial condition of the problem. Typical amplitudes of the perturbations are below $5 \%$ of the equilibrium value, and we performed several runs to test that the final result is insensitive to the values and signs of $\delta T$ and $\delta x$. We did not take higher values of $\delta$ since we believe that stronger initial fluctuations are of dubious physical origin. One may wonder if the isobaric assumption for the evolution is perhaps too severe and motions could develop; this requires a full hydrodynamical calculation. In this context we limit ourselves to solve the isobaric limit of the energy and ionization equations using a standard Runge-Kutta solver.

The most interesting questions concern the fate of unstable equilibria for which there are no corresponding stable temperatures for the same $p_{0} / \xi$. Important points are also the timescales of the formation of condensations usually originated by thermal instabilities. Before going into the detailed description of a representative case, we briefly summarize the main results of the nonlinear analysis.

1. The final fate of the nonlinear perturbation depends on the availability of stable states at the same pressure. If there are such states, the system will make a phase transition, as one could have expected.

2. When unstable states do not have other isobaric stable states (a situation not possible if ionization is considered constant) the system enters an oscillating, periodic state without approaching any stable value.

3. Oscillations, if present in the linear or nonlinear phase, cause a much slower transition to the final state.

For sake of clarity we will discuss separately unstable multi-phase (i.e., two or more isobaric equilibria of which at least one is stable) or unstable single-phase (i.e., one or more isobaric unstable equilibria). When the photon energy is high $(E \gg 13.6 \mathrm{eV})$ unstable equilibria are multi-phase; in the opposite case they are always single-phase (see Fig. 1). To illustrate in detail the results summarized above, we take an intermediate case: $E=40 \mathrm{eV}, Z=1$, which has both behaviors depending on the value of $p_{0} / \xi$. This being a transition case 
between monotonically growing curves of $p_{0} / \xi$ at low $E$ and wide multi-phase curves at high $E$, the shape of the curve is a bit peculiar and the range in which the $p_{0} / \xi$ curve allows for multi-phase equilibria is rather narrow. However, as discussed by Krolik et al.(1981) small ranges of $p_{0} / \xi$ involve wide range of gas densities and it is not so unlikely for the gas to lie in these regions. The equilibrium curve has been expanded for convenience in Fig. $5(a)$ and four points are marked in it for which nonlinear results are shown in Fig. 5(b) and discussed below. Numerical results refer to perturbations of equilibria with $N_{0} \rho=1 \mathrm{~cm}^{-3}$. The first point $P 1$ is a thermo-reactive stable equilibrium with $T_{0}=120 \mathrm{~K}, x_{0}=0.012$. In the linear phase the perturbation shows oscillations with $n_{i}=5 \times 10^{-6} \mathrm{yr}^{-1}$. These oscillations are clearly seen also during the nonlinear evolution before the perturbation is damped, a process which takes about $400 \tau_{h}=10^{7} \mathrm{yr}$.

- Single-phase equilibria. Equilibria $P 2$ and $P 3$ in Fig. $5(a)(E=40 \mathrm{eV})$ correspond to single-phase equilibria. For $P 2, T_{0}=150 \mathrm{~K}$ and $x_{0}=0.014$, and the perturbation is overstable in the linear phase (see also discussion in Sec. 3.2) with oscillation rate larger than the growth rate. For this reason, a large number of oscillations of growing amplitude is seen in T, $x, \rho$ before saturation takes place (Fig. 5(b)), as usually happens for thermoreactive unstable equilibria close to the unstable/stable transition region. After $t \sim 10^{3} \tau_{h}$ the system relaxes to a state of periodic oscillations of constant amplitude. Note that the ionization lags the temperature, as expected. Far from the unstable/stable transition region, as for the equilibrium point $P 3\left(T_{0}=1000 \mathrm{~K}, x_{0}=0.075\right)$ the value of $n_{i}$ is, in contrast, zero or rather small compared to the to the growth rate. Therefore for $P 3$ the perturbations grow to the saturated values in less than one oscillation period. After that moment, the evolution is qualitatively similar to that of $P 2$ but the oscillation is not centered around $T_{0}$, and the temperature spans the very large range $1000 \mathrm{~K} \lesssim T \lesssim 10,000 \mathrm{~K}$.

- Multi-phase equilibria. The point labelled P4 in Fig. 5 corresponds to an equilibrium temperature $T_{0}=2700 \mathrm{~K}$, and fractional ionization $x_{0}=0.18$. This equilibrium, which in the linear phase is thermo-reactively unstable with $n_{i}=0$, is obtained when $\xi \simeq$ $3 \times 10^{-14} \mathrm{~s}^{-1}$ for $N_{0} \rho=1 \mathrm{~cm}^{-3}$. The same value of $p_{0} / \xi$ corresponds also to the stable equilibrium point $T_{0}=8100 \mathrm{~K}, x_{0}=0.44$ but with a value of the volume density about 3 times smaller. The full nonlinear evolution of temperature and ionization fraction at constant $p_{0} / \xi$ is plotted in Fig. $5(b)$ as a function of time (normalized to the heating time $\left.\tau_{h}\right)$. The system reaches the other stable equilibrium after about $600 \tau_{h}=7.5 \times 10^{6} \mathrm{yr}$ through (nonlinear) oscillations. This time is much longer than the typical time scale that 
one would have calculated without the ionization, and, indeed, the system spends a long time in a time-dependent, nonequilibrium state. This retarding effect, due to ionization and already discussed for the linear stage, occurs very often and an additional, slightly different, case is shown in Fig. 6, corresponding to $E=100 \mathrm{eV}, Z=1$. Both temperatures, $T=150 \mathrm{~K}$ and $T=1000 \mathrm{~K}$, correspond to multi-phase equilibria; but when perturbed the first one shows oscillations in the linear phase whereas for the second $n_{i}=0$. It is clearly seen in the enlarged parts (bottom panels of Fig. 6) that the characteristic time for the phase transition is longer when oscillations are present.

To conclude, isobaric TR instabilities produce much slower phase transitions than the usual thermal instabilities. When no other stable equilibrium is available the medium spends long time intervals in nonequilibrium states characterized by nonlinear periodic oscillations of temperature, density and ionization fraction.

\section{SUMMARY AND IMPLICATIONS}

We have studied the acoustic and thermo-reactive instabilities in a diffuse ISM heated and ionized by an external radiation field, of mean photon energy $E$ and photoionization rate $\xi$, cooled by collisional excitation of metals and hydrogen lines. Thermo-reactive modes are found when the effects of time dependent ionization on the standard thermal instability are taken into account. The addition of the hydrogen recombination reaction to Field's classical treatment of thermal instability has several remarkable consequences especially when the ratio between the heat input and the ionization rate of the radiation field is rather small. In addition to the linear stability analysis we have investigated the nonlinear development of thermo-reactive modes. In general it would be interesting to extend this type of analysis to additional ionization and heating mechanisms (decaying neutrinos, dust grains, Alfven waves dissipation). For example, when present, dust grains may be important heating sources via the photoelectric effect. Since in this case the heat input depends linearly on the gas density, as for the photoionization heating, and the energy input per photoelectron is quite low $(\sim 1 \mathrm{eV})$, it is likely that some of the effects we found for low mean photon energies apply, if hydrogen remains the main free electron contributor. We summarize below the main results obtained in the range of temperatures of interest, $30 \mathrm{~K}<T<30,000 \mathrm{~K}$ and for metallicity in the range $0 \leq Z \leq 2$. 
The stability properties depend weakly on the mean photon energy absorbed: from Fig. 4 it is evident that as we penetrate inside the gas cloud, thus increasing $E$, the unstable modes remain the same. This means that the stability of the gas is mainly governed by the temperature rather than by the details of the radiation spectrum. Only for photon energies close to the Lyman limit and for intermediate values of $Z$ the response of the medium to perturbations may depend on $E$, and therefore edges of irradiated clouds can be sensibly different from their interiors.

In the absence of metals the gas is stable. A very small amount of metals induces non oscillatory unstable acoustic and thermo-reactive modes and, as in Field's analysis, the fastest growing instability is the isobaric thermal mode. Unstable equilibria require $p_{0} / \xi \gtrsim 10^{17} / Z \mathrm{~cm}^{-3} \mathrm{~K} \mathrm{~s}$, and growth times are short compared to the Hubble time only if $N_{0} \rho Z \gtrsim 10^{-3} \mathrm{~cm}^{-3}$. Low metallicity models can apply, for example, to low redshift Ly $\alpha$ clouds. In this case $p_{0} / \xi$ values are sufficiently low that only a stable warm phase could persist. According to Defouw's photoionization model (where no e-H cooling has been considered) these optically thin objects should instead be thermo-reactively unstable.

If the metal abundance is about half solar or higher the linear analysis shows that unstable modes often become oscillatory and ionization is a stabilizing agent, as explained in the previous Sections. Even though for column densities above $10^{18} \mathrm{~cm}^{-2}$, where the mean photon energy of the penetrating flux is larger than the hydrogen ionization energy, the range of thermally unstable temperatures is still wide, at low energies this range is considerably smaller and growth times are longer. Furthermore, a new type of situation arises in which the gas is thermo-reactively stable but unstable acoustic waves develop on large scales. While for $Z \simeq 0.5$ this occurs only for optically thin media, with $E-\chi \ll 100 \mathrm{eV}$, for larger $Z$ two regions are found which are only acoustically unstable, one in the cold phase at $T \sim 100 \mathrm{~K}$ and one in the warm phase at $T \sim 8000 \mathrm{~K}$. In places where there is no star formation for example, as in outer galactic HI disks, the development of these unstable acoustic waves could be the main source of motions in the gas. A nonlinear analysis of the evolution of the unstable acoustic waves is however necessary to assess whether shock waves develop and produce observational effects like non-thermal line broadening.

Another new and important feature introduced by the hydrogen recombination reaction is that the stability of isobaric thermo-reactive modes is no longer connected with the shape of the $p_{0} / \xi$ curve. This implies that there are unstable equilibria which cannot make an isobaric phase transition to any other stable state. The nonlinear analysis has shown that 
in this case the fate of the gas is to evolve towards a nonequilibrium state characterized by periodic, nonlinear oscillations of density, temperature and hydrogen ionization fraction. This effect is especially important for small heat input/ionization rate ratios, and for $Z>0.1$.

Promising objects to look for nonequilibrium oscillatory states are clouds located outside the optical disk of galaxies, or in the halo, like HVCs. These objects usually have low column density and therefore relatively low energy photons can penetrate. Their metallicity, though lower than solar, is still appreciably high (de Boer \& Savage 1984). Let us examine for example clouds with HI column density $N_{H} \simeq 10^{18} \mathrm{~cm}^{-2}$ irradiated by an extragalactic background flux with spectral index 1.5 as suggested by Sargent et al.(1979) and Madau (1992). The mean energy of ionizing photons at half total column density above the center is $E \sim 20 \mathrm{eV}$. Taking the value $p_{0} \simeq 100 \mathrm{~cm}^{-3} \mathrm{~K}$ as speculated by Ferrara \& Field (1994), with a flux intensity $I_{\nu} \sim 6 \times 10^{-24} \mathrm{erg} \mathrm{cm}^{-2} \mathrm{~s}^{-1} \mathrm{~Hz}^{-1}$ at the Lyman continuum, the corresponding ionizing rate is $\xi \simeq 2 \times 10^{-15} \mathrm{~s}^{-1}$ and $p_{0} / \xi \simeq 5 \times 10^{16} \mathrm{~cm}^{-3} \mathrm{~K}$ s. With these parameters the equilibrium temperature is $T_{0} \simeq 800 \mathrm{~K}$ and a single-phase thermo-reactive mode develops. The temperature in the nonlinear stages oscillates between 200 and 2500 $\mathrm{K}$ with a period of about $10^{8} \mathrm{yr}$, after a slightly longer nonlinear growth time. The average temperature corresponds to a FWHM of the HI line $\simeq 8 \mathrm{~km} \mathrm{~s}^{-1}$.

There are other environments where ionizing photon energies can be low enough that the nonequilibrium picture derived here can be expected, in contrast with the predictions of two-phase ISM models. Examples are high galactic latitude neutral hydrogen, where in fact average temperatures derived from observations correspond to thermally unstable equilibria (Verschuur \& Magnani 1994), and interfaces between low density HII regions and neutral gas. Even if the results derived here may not be directly applicable to bright HII regions where the important radiating ions are not those considered in this paper, photoionization models predict that low ionization stages of metals dominate the cooling in the diffuse ionized layer responsible for the pervasive Galactic $\mathrm{H} \alpha$ emission (see for example Domgörgen \& Mathis 1994). When photons from the stars penetrate through interstellar "tunnels", the gas can be in a nonequilibrium state involving isobaric periodic oscillations, or show motions due to unstable acoustic waves, depending upon local values of optical depth and thermal pressure. 
TABLE 1

SYMBOLS USED FREQUENTLY

\begin{tabular}{|c|c|}
\hline $\begin{array}{l}\text { Symbol } \\
(1)\end{array}$ & $\begin{array}{l}\text { Meaning } \\
(2)\end{array}$ \\
\hline$\rho, T, \mathbf{v}, p, x$ & $\begin{array}{l}\text { Density, temperature, velocity, pressure and ionization fraction; } \\
\text { subscript } 0 \text { indicates unperturbed value, } 1 \text { indicates perturbation }\end{array}$ \\
\hline$\underset{\mathrm{Z}}{\gamma}, N_{0}, k_{B}$ & $\begin{array}{l}\text { Ratio of specific heats, Avogadro number, Boltzmann constant } \\
\text { Metallicity }\end{array}$ \\
\hline $\mathcal{L}, \mathcal{L}_{T}, \mathcal{L}_{\rho}, \mathcal{L}_{x}$ & Generalized heat-loss function per unit mass and its $\mathrm{T}$ - $\rho$ - and $x$-derivatives \\
\hline$I, I_{T}, I_{\rho}, I_{x}$ & Generalized ionization-recombination function per unit mass and its $T-\rho$ - ar \\
\hline$\Lambda_{H Z}, \Lambda_{e Z}, \Lambda_{e H}, \Lambda_{e H^{+}}$ & Cooling losses due hydrogen-metal, electron-metal, electron-hydrogen, electr \\
\hline & Speed of sound \\
\hline$n, k, \lambda$ & Growth rate, wavenumber, and wavelength of perturbation \\
\hline$n_{r}, n_{i}$ & Real and imaginary part of the growth rate $n$ \\
\hline$n_{m}, k_{m}$ & Largest real part of the growth rates, and value of $k$ for which this maximun \\
\hline$\xi, E$ & Photoionization rate and mean photon energy of the incident radiation at a \\
\hline$\phi, E_{h}$ & Number of secondary electrons and thermal heat released per photoionizatio \\
\hline$\tau_{h}, \tau_{d}$ & Heating time, $\tau_{h} \equiv(5 / 2) k_{B} T / \xi E_{h}$, dynamical time, $\tau_{d} \equiv 1 / c_{s} k$ \\
\hline$\tau_{T}, \tau_{\rho}, \tau_{x}$ & $\begin{array}{l}\text { Characteristic cooling times for the perturbed gas, where: } \\
\tau_{T} \equiv(3 / 2) k_{B} T(1+x) N_{0} /\left|T \mathcal{L}_{T}\right|, \tau_{\rho} \equiv(3 / 2) k_{B} T(1+x) N_{0} /\left|\rho \mathcal{L}_{\rho}\right|, \tau_{x} \equiv(3 / 2)\end{array}$ \\
\hline $\bar{\tau}_{T}, \bar{\tau}_{\rho}, \bar{\tau}_{x}$ & Characteristic reactive times for the perturbed gas, where: \\
\hline$K_{T}, K_{\rho}, K_{x}$ & $\begin{array}{l}\bar{\tau}_{T} \equiv(1+x) N_{0} /\left|T I_{T}\right|, \bar{\tau}_{\rho} \equiv(1+x) N_{0} /\left|\rho I_{\rho}\right|, \bar{\tau}_{x} \equiv(1+x) N_{0} /\left|(1+x) I_{x}\right| \\
\text { Characteristic wavenumbers associated with cooling processes: } \\
K_{T}=\left(\mathcal{L}_{T} /\left|\mathcal{L}_{T}\right|\right) / \tau_{T} c_{s}, K_{\rho}=\left(\mathcal{L}_{\rho} /\left|\mathcal{L}_{\rho}\right|\right) / \tau_{\rho} c_{s}, K_{x}=\left(\mathcal{L}_{x} /\left|\mathcal{L}_{x}\right|\right) / \tau_{x} c_{s}\end{array}$ \\
\hline$J_{T}, J_{\rho}, J_{x}$ & $\begin{array}{l}\text { Characteristic wavenumbers associated with reactive processes: } \\
J_{T}=\left(I_{T} /\left|I_{T}\right|\right) / \tau_{T} c_{s}, J_{\rho}=\left(I_{\rho} /\left|I_{\rho}\right|\right) / \tau_{\rho} c_{s}, J_{x}=\left(I_{x} /\left|I_{x}\right|\right) / \tau_{x} c_{s}\end{array}$ \\
\hline$\Theta$ & Inverse temperature $\equiv 157900 / T$ \\
\hline$\gamma_{c} \tau_{\tilde{\gamma}}$ & Collisional ionization rate $\quad \tilde{v}=v /(3 / 2) k_{0} T$ \\
\hline$\chi, \chi^{t h}$ & Hydrogen ionization potential, $\tilde{\chi} \equiv \chi /(3 / 2) k_{B} T$ \\
\hline$\alpha, E^{t h}$ & Total radiative recombination coefficient and thermal energy lost by recombi \\
\hline
\end{tabular}




\section{ACKNOWLEDGMENTS}

We are grateful to J. M. Dickey, E.E. Salpeter and especially to G. B. Field for interesting discussions and comments on the subject of this paper. E.C. acknowledges the Agenzia Spaziale Italiana for financial support.

\section{REFERENCES}

Aleksandrov, A.D., Kolmogorov, A. N. \& Lavrent'ev, M. A. 1963, Mathematics: Its Content, Methods, and Meaning, (MIT Press: Cambridge)

Black, J. H. 1981, MNRAS, 197, 555

Charlton, J. C, Salpeter, E. E. \& Hogan C. J. 1993, ApJ, 402, 493

Collin-Souffrin, S. 1990, in New Windows to the Universe, vol. 2, ed. F. Sanchez \& M. Vasquez (Cambridge: Univ Press), 235

Corbelli, E. \& Salpeter, E. E. 1993a, ApJ, 419, 94

Corbelli, E. \& Salpeter, E. E. 1993b, ApJ, 419, 104

Dalgarno, A. \& McCray, R. A. 1972, ARA\&A, 10, 375

de Boer, K. S. \& Savage, B. D. 1984, ApJ, 136, L7

Defouw, R. J. 1970, ApJ, 161, 55

Dickey, J. M., Murray, H. M. \& Helou, G. 1990, ApJ, 352, 522

Domgörgen, H. \& Mathis J. S. 1994, ApJ, 428, 647

Dove J. B. \& Shull, J. M. 1994, ApJ, 423, 196

Ferrara, A. \& Field, G. B. 1994, ApJ 423, 665

Field, G. B. 1965, ApJ, 142, 531

Field, G. B., Goldsmith, D. W. \& Habing, H. J. 1969, ApJ, 155, L149

Flannery, B. P. \& Press, W. H. 1979, ApJ, 231, 688

Goldsmith, G. W. 1970, ApJ, 161, 41

Ibañez, M. H. \& Parravano A. 1983, ApJ, 275, 181

Ikeuchi S. \& Turner 1991, ApJ, 381, L1

Krolik, J. H., McKee, C. F., \& Tarter, C. B. 1981, ApJ, 249, 422

Kulkarni, V. S. \& Fall, S. M 1993, ApJ, 413, L63

Kwan, J. Y. \& Krolik, J. H. 1981, ApJ, 250, 468

Launay, M. \& Roueff, E. 1977, A\&A, 56, 289

Madau, P. 1992, ApJ, 389, L1

Maloney, P. 1993, ApJ, 414, 14

McKee, C. F. \& Ostriker, J. P. 1977, ApJ, 218, 148

Miralda-Escudé, J. \& Ostriker, J. P. 1992, ApJ, 392, 15

Reynolds, R. J. 1993, in 3rd Annual Maryland Meeting, Back to the Galaxy, (Washington: NASA), 156

Sargent, W. L. W., Young, P. J., Boksenberg, A., Carswell, R. F., \& Whelan, J. A. J. 1979, ApJ, 230, 49

Shull, J. M., \& Van Steenberg, M. E. 1985, ApJ, 298, 268

Songaila, A., Bryant, W. \& Cowie, L. L. 1989, ApJ, 345, L71

Yoneyama, T. 1973, PASJ, 25, 349

Verschuur, G. L. \& Magnani, L. 1994, AJ, 107, 287 


\section{FIGURE CAPTIONS}

Figure 1. Equilibrium curves of $p_{0} / \xi$ for four values of the metallicity $Z . p_{0}$ is in units of $\mathrm{cm}^{-3} \mathrm{~K}$ and $\xi$ is the photoionization rate in $\mathrm{s}^{-1}$. For each value of $Z$ we show the equilibrium curves for three energies: in each panel the bottom curve is for $E=15 \mathrm{eV}$, the middle curve is for $E=40 \mathrm{eV}$ and the top curve is for $E=100 \mathrm{eV}$. Dashed lines denote unstable equilibria; solid lines denote stable equilibria.

Figure 2. Positive real part of growth rates $n_{r}$ as function of the wavenumber $k$ for three equilibrium temperatures relative to $E=100 \mathrm{eV}, Z=1$, and $N_{0} \rho=1 \mathrm{~cm}^{-3}$. For $T_{0}=120 \mathrm{~K}$ we plot the growth rate multiplied by a factor of 10 . For this temperature the equilibrium is stable at large $k$ while for $T_{0}=160 \mathrm{~K}$ and $T_{0}=200 \mathrm{~K}$ there is an unstable mode at all wavenumbers. The maximum growth rate for $T_{0}=200 \mathrm{~K}$ is for $k \rightarrow \infty$.

Figure 3. In $(a)$ the largest positive real part of growth rates, $n_{m}$, is plotted as a function of the equilibrium temperature $T_{0}$. In $(b)$ we plot the wavenumber $k_{m}$ for which the largest growth rate has been found. For some values of $n_{m}, k_{m} \rightarrow \infty$; in this case $k_{m}$ has not been plotted. We have used $N_{0} \rho=1$ $\mathrm{cm}^{-3}$. Each panel in $(a)$ or $(b)$ refers to a different photon energy $E(\mathrm{eV})$. The continuous curves are for metallicity $Z=1$, the small dashed curves for $Z=0.5$ and the dotted-dashed curve for $Z=10^{-3}$.

Figure 4. Unstable regions in the plane $E-T$ for various values of the metallicity $Z$. In the dotted regions there are unstable modes only for $k<k_{m}$ (acoustic waves). In the dashed regions equilibria have unstable modes for any $k$ (they are both acoustically and thermo-reactively unstable). The dashed curves at the bottom and left hand side of each panel limit the possible equilibrium values of $T_{0}$ for each value of the photon energy $E$.

Figure 5. (a) part of equilibrium curve $p_{0} / \xi-T_{0}$ for $E=40 \mathrm{eV}$ and $Z=1$. The dashed-dotted lines refers to equilibria which have unstable thermo-reactive modes. For the four equilibrium temperatures marked on the curve we plot in $(b)$ the nonlinear evolution of the temperature and ionization fraction perturbations in the isobaric regime. Continuous curves refer to $\delta T / T_{0}$, dotted curves to $\delta x / x_{0}$ as function of time (normalized to the heating time $\left.\tau_{H}\right)$. The density is set to $N_{0} \rho=1 \mathrm{~cm}^{-3}$.

Figure 6. Nonlinear evolution of temperature and ionization fraction perturbations in the isobaric regime for two multi-phase equilibria in the case $E=100$ $\mathrm{eV}, Z=1$, and $N_{0} \rho=1 \mathrm{~cm}^{-3}$. Continuous curves refer to $\delta T / T_{0}$, dotted curve to $\delta x / x_{0}$ as function of time (normalized to the heating time $\tau_{H}$ ). Bottom panels show enlarged portions of top panels. 